Homology, Homotopy and Applications, vol.12(2), 2010, pp.321-334

\title{
CYCLIC HOMOLOGY VIA DERIVED FUNCTORS
}

\author{
GURAM DONADZE, NICK INASSARIDZE AND MANUEL LADRA \\ (communicated by Graham Ellis)

\begin{abstract}
The cyclic, periodic cyclic and negative cyclic homologies of associative algebras are fitted into the context of cotriple homology of Barr and Beck. As applications of these results, an axiomatic description of the cyclic homology theory and the Hopf type formulas in the sense of Brown-Ellis are given.
\end{abstract}

\section{Introduction}

One powerful tool of simplicial algebra is the notion of cotriple derived functors. It has been applied to the simplicial group approach to algebraic $K$-theory worked out by Keune [15] and Swan [20] and further developed in non-abelian homological algebra by $\mathrm{H}$. Inassaridze $[\mathbf{1 2}]$ and others.

On the other hand, in the works of Barr and Beck $[\mathbf{1}, \mathbf{2}, \mathbf{3}, \mathbf{4}]$, Eilenberg-MacLane group (co)homology, Hochschild and Shukla (co)homology of associative algebras, André-Quillen (co)homology of commutative algebras, Cartan-Eilenberg and Chevalley-Eilenberg (co)homology of Lie algebras have been described in terms of nonabelian derived functors as cotriple (co)homology (see also [8]). Namely, in all these cases, there is a cotriple on the category in question that comes from the composite of the underlying and the free functor. Each of the (co)homologies above are computed by applying the derivations functor for the cohomological case and the functor obtained by tensoring a module on the functor of differentials for the homological case to the cotriple resolution and then taking the (co)homology of the resulted complex.

This paper is concerned with the description of an analogous philosophy in cyclic homology. Our main results, filling the gap in the subject, present the cyclic, periodic cyclic and negative cyclic homologies of (non-unital) associative algebras over a commutative ring $k$ containing the field $\mathbb{Q}$ of rational numbers as cotriple derived functors. As applications of these results, we then obtain an axiomatic description and the Hopf type formulas in the sense of Brown and Ellis [5, 9] (see also [10] for the historical note about the subject) for cyclic homology which are interesting in their own right.

The authors were supported by MICINN grant MTM2009-14464-C02 (European FEDER support included) and by Xunta de Galicia grant Incite09207215PR. The first two authors were also partially supported by Volkswagen Foundation Ref.: I/84 328.

Received June 1, 2010, revised August 10, 2010; published on November 4, 2010.

2000 Mathematics Subject Classification: 16E40, 18G10, 18G50.

Key words and phrases: cyclic homology, periodic cyclic homology, negative cyclic homology, cotriple derived functor.

This article is available at http://intlpress.com/HHA/v12/n2/a10

Copyright (C) 2010, International Press. Permission to copy for private use granted. 
Some of the results presented in this paper have been announced in [14].

The outline of the paper is as follows: In Section 2, we briefly recall the necessary background information about cotriple derived functors and give some auxiliary assertions that will be used in main results. We begin Section 3 by giving a long exact sequence relating Hochschild homology and the cotriple derived functors of the zero and first Hochschild homology functors (Proposition 3.1). We revisit the old result of Feigin and Tsygan [11] given for algebras over a field of characteristic zero. Generalising it for algebras, which are flat as modules over a commutative ring $k$ containing the field $\mathbb{Q}$ of rational numbers, we show that the cyclic homology is isomorphic to the derived functors of the additive abelianisation functor (Theorem 3.5). We end this section by giving an axiomatic description and the generalised Hopf type formulas for the cyclic homology of algebras (Proposition 3.8 and Theorem 3.10). In Section 4, we describe the periodic cyclic and negative cyclic homology theories as derived functors (Theorems 4.2 and 4.3).

\section{Notation and conventions}

Let $k$ denote a fixed commutative ring with unit and $\mathbb{Q}$ the field of rational numbers. All tensor products are over $k$. Moreover, $A^{\otimes n}=A \otimes \cdots \otimes A, n$ factors. Modules are $k$-modules and their category is denoted by Mod, while $\mathbf{C o m p}_{\geqslant 0}$ is the category of non-negatively graded complexes of modules. For any bicomplex of modules $\mathfrak{M}$, let $\operatorname{Tot}(\mathfrak{M})_{*}$ denote the unbounded complex given by

$$
\operatorname{Tot}(\mathfrak{M})_{n}=\prod_{p+q=n} M_{p, q}, n \in \mathbb{Z}
$$

and called the total complex of $\mathfrak{M}$. Algebras are (non-unital) associative algebras over $k$ and their category is denoted by Alg. The term free algebra means a free (non-unital) algebra over some module. Ideals are always two-sided. For any algebra $A$ and ideals $I_{1}$ and $I_{2}$, let $\left[I_{1}, I_{2}\right]$ denote the module of additive commutators, i.e., the submodule of $A$ generated by the elements $\left[\mathfrak{i}_{1}, \mathfrak{i}_{2}\right]=\mathfrak{i}_{1} \mathfrak{i}_{2}-\mathfrak{i}_{2} \mathfrak{i}_{1}$ for $\mathfrak{i}_{1} \in I_{1}, \mathfrak{i}_{2} \in I_{2}$.

\section{Preliminaries on derived functors}

We assume that the reader is familiar with cotriples, projective classes and derived functors. We give just a brief introduction to these aspects for the category of algebras. A fuller account of cotriple derived functors is given in $[\mathbf{3}, \mathbf{1 2}]$. Note that in some categorical and homological literature the term 'comonad' is used instead of the term 'cotriple'.

Let $(\mathcal{F}, \tau, \delta)$ denote the cotriple in $\mathbf{A l g}$ constructed in the following way: let $\mathcal{F}: \mathbf{A l g} \rightarrow \mathbf{A l g}$ be the endofunctor defined as follows: for an object $A$ of $\mathbf{A l g}$, let $\mathcal{F}(A)$ denote the free algebra on the underlying module $A$; for a morphism $\alpha: A \rightarrow A^{\prime}$ of Alg, let $\mathcal{F}(\alpha)$ be the canonical algebra homomorphism from $\mathcal{F}(A)$ to $\mathcal{F}\left(A^{\prime}\right)$ induced by $\alpha$. Let $\tau: \mathcal{F} \rightarrow 1_{\mathrm{Alg}}$ be the obvious natural transformation and $\delta: \mathcal{F} \rightarrow \mathcal{F}^{2}$ the natural transformation induced for every $A \in \mathbf{A l g}$ by the natural inclusion of modules $A \hookrightarrow \mathcal{F}(A)$. 
Let $A \in \mathbf{A l g}$; then there is an augmented simplicial object in the category $\mathbf{A l g}$

$$
\cdots \underset{\dot{\rightarrow}}{\rightarrow} \mathcal{F}_{n}(A) \underset{d_{n}^{n}}{\stackrel{d_{0}^{n}}{\vec{a}}} \cdots \underset{d_{2}^{2}}{\stackrel{d_{0}^{2}}{\rightarrow}} \mathcal{F}_{1}(A) \underset{d_{1}^{1}}{\stackrel{d_{0}^{1}}{\rightarrow}} \mathcal{F}_{0}(A) \stackrel{d_{0}^{0}}{\rightarrow} A,
$$

written as $\left(\mathcal{F}_{*}(A), d_{0}^{0}, A\right)$, where $\mathcal{F}_{n}(A)=\mathcal{F}^{n+1}(A)=\mathcal{F}\left(\mathcal{F}^{n}(A)\right), d_{i}^{n}=\mathcal{F}^{i}\left(\tau_{\mathcal{F}^{n-i}}\right)$, $s_{i}^{n}=\mathcal{F}^{i}\left(\delta_{\mathcal{F}^{n-i}}\right), 0 \leqslant i \leqslant n$, and which is called the $\mathcal{F}$-cotriple resolution of $A$ (see also $[\mathbf{2 0}])$.

The following lemma will be useful.

Lemma 2.1. Let $A$ be an algebra.

(i) $\left(\mathcal{F}_{*}(A), d_{0}^{0}, A\right)$ is acyclic.

(ii) If $A$ is flat as a module, then $\left(\mathcal{F}_{*}(A)^{\otimes n}, d_{0}^{0}{ }^{\otimes n}, A^{\otimes n}\right), n \geqslant 1$, is acyclic.

Proof of (i) The proof of (i) is well known.

Proof of (ii). Proceeding by induction, we suppose that

$$
\left(\mathcal{F}_{*}(A)^{\otimes(n-1)}, d_{0}^{0 \otimes(n-1)}, A^{\otimes(n-1)}\right)
$$

is acyclic.

It is clear that $\left(\mathcal{F}_{*}(A)^{\otimes n}, d_{0}^{0}{ }^{\otimes n}, A^{\otimes n}\right)$ is the diagonal of the augmented bisimplicial module obtained by tensoring dimensionwise $\left(\mathcal{F}_{*}(A)^{\otimes(n-1)}, d_{0}^{0 \otimes(n-1)}, A^{\otimes(n-1)}\right)$ and $\left(\mathcal{F}_{*}(A), d_{0}^{0}, A\right)$. Since $A$ is flat as a module, it is easy to see that $\mathcal{F}_{p}(A)^{\otimes l}, p \geqslant 0$, $l \geqslant 1$, is also flat as a module. Hence for a fixed $p$ the (vertical) homology is

$$
H_{q}\left(\mathcal{F}_{p}(A)^{\otimes(n-1)} \otimes \mathcal{F}_{*}(A)\right)=0, q>0
$$

and

$$
H_{0}\left(\mathcal{F}_{p}(A)^{\otimes(n-1)} \otimes \mathcal{F}_{*}(A)\right)=\mathcal{F}_{p}(A)^{\otimes(n-1)} \otimes A .
$$

Using the same argument, we get

$$
H_{p}\left(\mathcal{F}_{*}(A)^{\otimes(n-1)} \otimes A\right)=0, p>0,
$$

and

$$
H_{0}\left(\mathcal{F}_{*}(A)^{\otimes(n-1)} \otimes A\right)=A^{\otimes n} .
$$

Now the respective Quillen spectral sequence [18] (cf. [12]) yields that

$$
\left(\mathcal{F}_{*}(A)^{\otimes n}, d_{0}^{0}{ }^{\otimes n}, A^{\otimes n}\right)
$$

is acyclic.

Let $T: \mathbf{A l g} \rightarrow \operatorname{Mod}$ be a covariant functor. Then the $n$-th left derived functor

$$
L_{n}^{\mathcal{F}} T: \text { Alg } \rightarrow \text { Mod, } \quad n \geqslant 0,
$$

relative to the cotriple $\mathcal{F}$, is defined by

$$
L_{n}^{\mathcal{F}} T(A)=H_{n}\left(T\left(\mathcal{F}_{*}(A)\right)\right) \quad \text { and } \quad L_{n}^{\mathcal{F}} T(\alpha)=H_{n}\left(T\left(\mathcal{F}_{*}(\alpha)\right)\right)
$$

for any object $A \in \mathbf{A l g}$ and any morphism $\alpha \in \mathbf{A l g}$. 
Suppose now that $\Phi: \mathbf{A l g} \rightarrow \mathbf{C o m p}_{\geqslant 0}$ is a covariant functor. Thus, for any object $A \in \mathbf{A l g}$, we have a complex:

$$
\Phi(A) \equiv \Phi_{0}(A) \stackrel{d_{1}}{\longleftarrow} \Phi_{1}(A) \stackrel{d_{2}}{\longleftarrow} \Phi_{2}(A) \stackrel{d_{3}}{\longleftarrow} \cdots
$$

Define the $\Phi$-homology of $A, H_{*}^{\Phi}(A)$, by

$$
H_{n}^{\Phi}(A)=H_{n}(\Phi(A)), \quad n \geqslant 0 .
$$

The following assertion will be needed in the sequel.

Proposition 2.2. Let $\Phi: \mathbf{A l g} \rightarrow \mathbf{C o m p}_{\geqslant 0}$ be a functor and $A$ an algebra, subject to the following conditions:

(i) the augmented simplicial module $\left(\Phi_{n}\left(\mathcal{F}_{*}(A)\right), \Phi_{n}\left(d_{0}^{0}\right), \Phi_{n}(A)\right)$ is acyclic for $n \geqslant 0$;

(ii) $H_{n}^{\Phi}\left(\mathcal{F}_{j}(A)\right)=0$ for $j \geqslant 0, n>0$,

where $\left(\mathcal{F}_{*}(A), d_{0}^{0}, A\right)$ is the $\mathcal{F}$-cotriple resolution of $A$. Then there is a natural isomorphism

$$
H_{n}^{\Phi}(A) \cong L_{n}^{\mathcal{F}} H_{0}^{\Phi}(A), \quad n \geqslant 0
$$

Proof. Applying the functor $\Phi$ dimensionwise to the simplicial algebra $\mathcal{F}_{*}(A)$, we arrive at the following bicomplex $\mathfrak{M}$ :

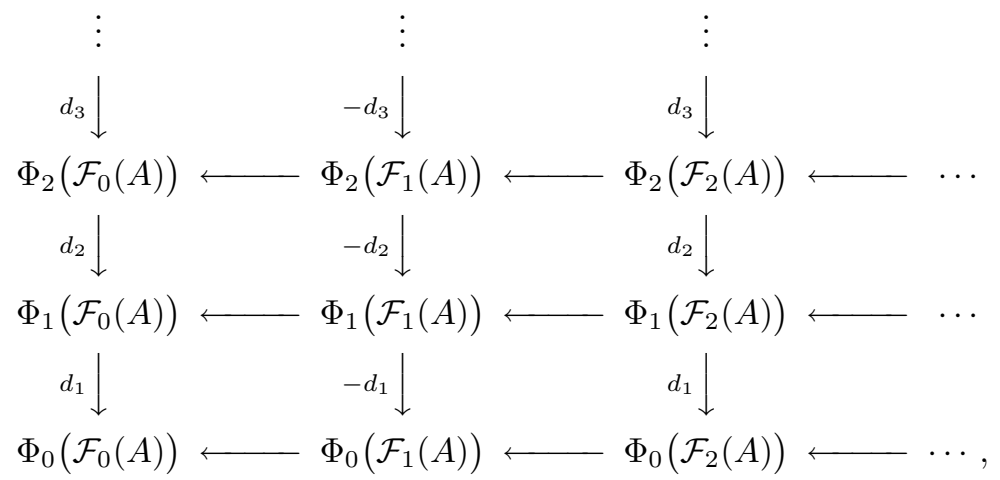

where $\left(\mathcal{F}_{*}(A), d_{0}^{0}, A\right)$ is the $\mathcal{F}$-cotriple resolution of $A$ and the horizontal differentials are obtained by taking alternating sums. Then by (i), we have

$$
H_{q}^{v} H_{p}^{h}(\mathfrak{M})=0, p>0, q \geqslant 0 \quad \text { and } \quad H_{q}^{v} H_{0}^{h}(\mathfrak{M})=H_{q}^{\Phi}(A), q \geqslant 0 .
$$

On the other hand, using (ii), we get

$$
H_{p}^{h} H_{q}^{v}(\mathfrak{M})=0, p \geqslant 0, q>0 \quad \text { and } \quad H_{p}^{h} H_{0}^{v}(\mathfrak{M})=L_{p}^{\mathcal{F}} H_{0}^{\Phi}(A), p \geqslant 0 .
$$

Now the bicomplex spectral sequence argument completes the proof.

\section{Cyclic homology revisited}

In this section we revisit the Hochschild and cyclic homologies of algebras, using methods of non-abelian homological algebra (we refer here to $[\mathbf{4}, \mathbf{1 2}, \mathbf{2 1}]$ ). We give 
several presentations of the cyclic homology of algebras as cotriple derived functors. Then we give an axiomatic description of the cyclic homology of algebras. We end the section by giving a decomposition of the cyclic homology of algebras as formulas of Hopf type all components of which are coming from any free exact $n$-presentation of a given algebra.

Let $A$ denote an algebra. Then the standard bar, $C^{\text {bar }}(A)$, and Hochschild, $C(A)$, complexes of $A$ have the form $C_{n}^{\text {bar }}(A)=C_{n}(A):=A^{\otimes(n+1)}$, where the boundary operator of the bar complex is given by

$$
b^{\prime}\left(\mathfrak{a}_{0} \otimes \cdots \otimes \mathfrak{a}_{n}\right)=\sum_{i=0}^{n-1}(-1)^{i}\left(\mathfrak{a}_{0} \otimes \cdots \otimes \mathfrak{a}_{i} \mathfrak{a}_{i+1} \otimes \cdots \otimes \mathfrak{a}_{n}\right),
$$

while the Hochschild boundary is given by

$$
b\left(\mathfrak{a}_{0} \otimes \cdots \otimes \mathfrak{a}_{n}\right)=b^{\prime}\left(\mathfrak{a}_{0} \otimes \cdots \otimes \mathfrak{a}_{n}\right)+(-1)^{n}\left(\mathfrak{a}_{n} \mathfrak{a}_{0} \otimes \mathfrak{a}_{1} \otimes \cdots \otimes \mathfrak{a}_{n-1}\right) .
$$

The Hochschild homology of $A, H H_{*}(A)$, is defined to be the homology of the complex Tot $\left(C C^{\{2\}}(A)\right)_{*}$, where $C C^{\{2\}}(A)$ denotes the bicomplex obtained through deleting all columns whose indices are $\leqslant-1$ or $\geqslant 2$ of the bicomplex $C P(A)$ :

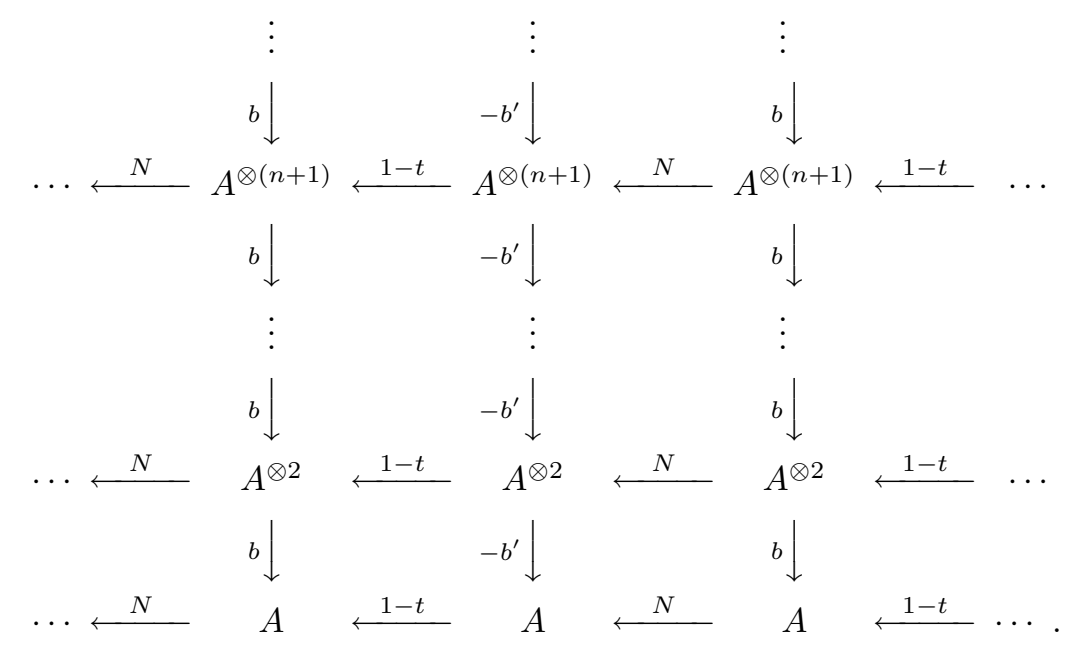

Here $t: A^{\otimes(n+1)} \rightarrow A^{\otimes(n+1)}, n \geqslant 0$ denotes the cyclic operator given by $t\left(\mathfrak{a}_{0} \otimes \cdots\right.$ $\left.\otimes \mathfrak{a}_{n}\right)=(-1)^{n}\left(\mathfrak{a}_{n} \otimes \mathfrak{a}_{0} \otimes \cdots \otimes \mathfrak{a}_{n-1}\right)$, and $N: A^{\otimes(n+1)} \rightarrow A^{\otimes(n+1)}$ denotes the operator defined by $N=1+t+t^{2}+\cdots+t^{n}$. By deleting all negatively indexed columns in $C P(A)$, we get the cyclic bicomplex $C C(A)$ and the homology of the complex Tot $(C C(A))_{*}$ is called the cyclic homology of $A$.

Note that, for a given free algebra $F$, the Hochschild homology $H H_{n}(F)=0$, $n \geqslant 2$. Moreover, if $k$ contains $\mathbb{Q}$ then the cyclic homology $H C_{n}(F)=0, n \geqslant 1[\mathbf{1 7}$, Proposition 5.4] (see also [16, Chapter 3]). We also have to mention that

$$
H H_{0}(A)=H C_{0}(A)=\mathcal{A} b(A)
$$

where $\mathcal{A} b: \mathbf{A l g} \rightarrow \mathbf{M o d}$ is the additive abelianisation functor given by $\mathcal{A} b(A)$ $=A /[A, A]$ for $A \in \mathbf{A l g}$. 
The following proposition gives a relationship between the Hochschild homology and the derived functors of the additive abelianisation functor.

Proposition 3.1. Let $A$ be an algebra, which is flat as a module. Then there is a long exact sequence

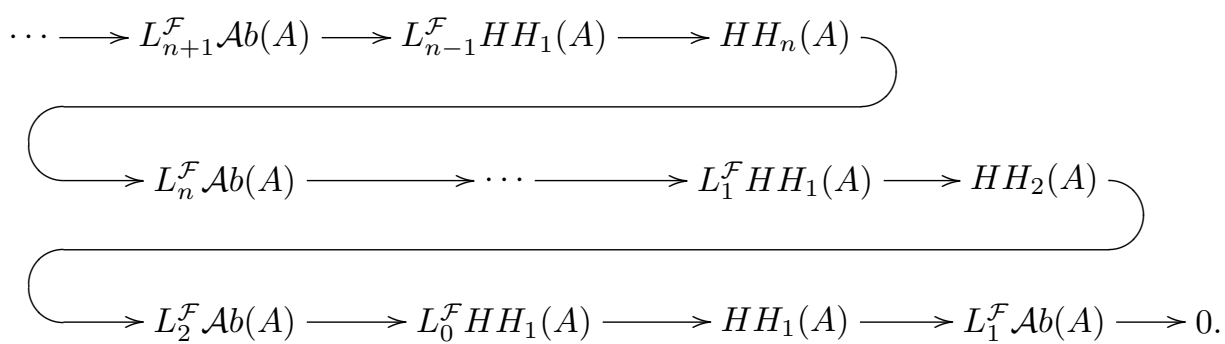

Proof. Consider the $\mathcal{F}$-cotriple resolution $\left(\mathcal{F}_{*}(A), d_{0}^{0}, A\right)$ of $A$. Applying the functor $C C^{\{2\}}$ dimensionwise to the simplicial algebra $\mathcal{F}_{*}(A)$, we arrive at the following bicomplex $\mathfrak{M}$ :

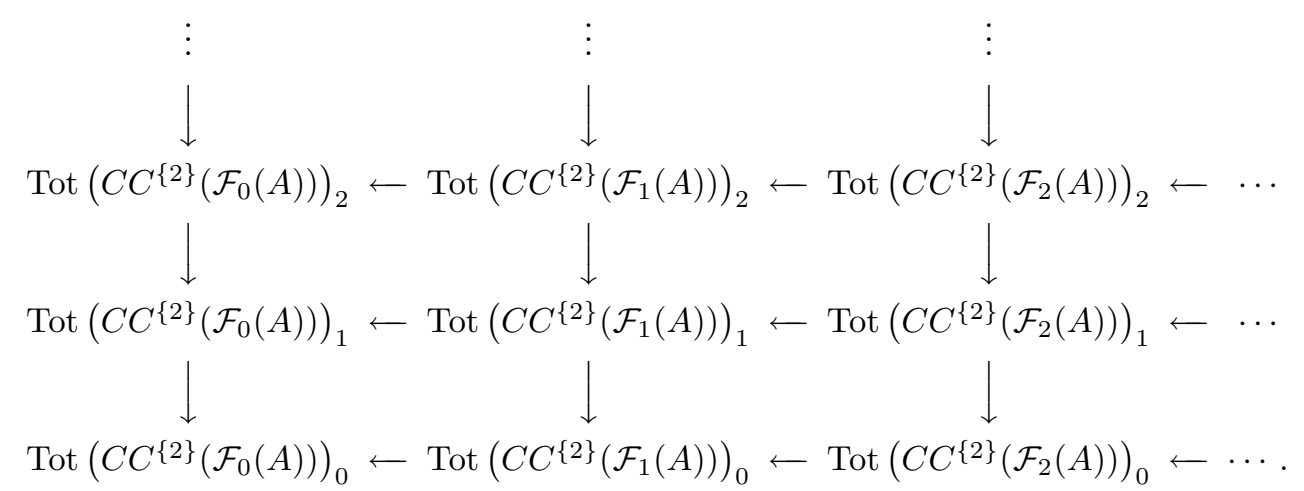

Since for any fixed $q$ the (horizontal) complex has the form $\mathcal{F}_{*}(A)^{\otimes(q+1)} \oplus \mathcal{F}_{*}(A)^{\otimes q}$, it follows from Lemma 2.1 that its homology is

$$
H_{n}\left(\operatorname{Tot}\left(C C^{\{2\}}\left(\mathcal{F}_{*}(A)\right)\right)_{q}\right)=0 \quad \text { for } n>0,
$$

and

$$
H_{0}\left(\operatorname{Tot}\left(C C^{\{2\}}\left(\mathcal{F}_{*}(A)\right)\right)_{q}\right)=A^{\otimes(q+1)} \oplus A^{\otimes q} .
$$

Therefore, $H_{n}\left(\operatorname{Tot}(\mathfrak{M})_{*}\right)=H H_{n}(A)$. On the other hand, for a fixed $p$ the (vertical) homology is

$$
H_{n}\left(\operatorname{Tot}\left(C C^{\{2\}}\left(\mathcal{F}_{p}(A)\right)\right)_{*}\right)=H H_{n}\left(\mathcal{F}_{p}(A)\right), n \geqslant 0
$$

A consequence of these computations is the existence of a convergent spectral sequence

$$
E_{p q}^{1}=H H_{q}\left(\mathcal{F}_{p}(A)\right) \Longrightarrow H H_{p+q}(A),
$$

but the Hochschild homology $E_{p q}^{1}=H H_{q}\left(\mathcal{F}_{p}(A)\right)=0$ for $q \geqslant 2, p \geqslant 0$ (see above). Moreover, $E_{p q}^{2}=L_{p}^{\mathcal{F}} H H_{q}(A)$ for $q=0,1, p \geqslant 0$. Then the degenerated spectral sequence $E_{p q}^{2}$ yields the result. 
Let $A$ be an algebra and $I$ an ideal in $A$. Let $I \otimes A$ denote the module given by the quotient $I \otimes A=(I \otimes A) / J$, where $J$ is the submodule of $I \otimes A$, generated by the elements $\mathfrak{i} \mathfrak{a} \otimes \mathfrak{a}^{\prime}-\mathfrak{i} \otimes \mathfrak{a} \mathfrak{a}^{\prime}+\mathfrak{a}^{\prime} \mathfrak{i} \otimes \mathfrak{a}$ and $\mathfrak{i} \otimes \mathfrak{i}^{\prime}+\mathfrak{i}^{\prime} \otimes \mathfrak{i}$ for $\mathfrak{i}, \mathfrak{i}^{\prime} \in I, \mathfrak{a}, \mathfrak{a}^{\prime} \in A$. It is easy to see that we have a homomorphism of modules

$$
I \otimes A \stackrel{\widetilde{b}}{\longrightarrow} I \quad \text { given by } \widetilde{b}(\mathfrak{i} \otimes \mathfrak{a})=\mathfrak{i} \mathfrak{a}-\mathfrak{a} \mathfrak{i} .
$$

It is well known that there is an exact sequence

$$
0 \rightarrow H C_{1}(A) \rightarrow A \bigotimes A \stackrel{\tilde{b}}{\rightarrow} A \rightarrow H C_{0}(A) \rightarrow 0 .
$$

Lemma 3.2. Let $0 \rightarrow I \stackrel{\alpha}{\rightarrow} A \stackrel{\beta}{\rightarrow} A^{\prime} \rightarrow 0$ be an algebra extension. Then there is an exact sequence of modules

$$
I \otimes A \stackrel{\alpha \otimes 1}{\longrightarrow} A \otimes A \stackrel{\beta \otimes \beta}{\longrightarrow} A^{\prime} \otimes A^{\prime} \longrightarrow 0 .
$$

Proof. Straightforward from the well-known fact that there is an exact sequence of modules

$$
(I \otimes A) \times(A \otimes I) \stackrel{\omega}{\longrightarrow} A \otimes A \stackrel{\beta \otimes \beta}{\longrightarrow} A^{\prime} \otimes A^{\prime} \longrightarrow 0
$$

with $w=(\alpha \otimes 1) \times(1 \otimes \alpha)$ and from direct observation.

Later, using Theorem 3.5, the following result could be viewed as a description of cyclic homology via derived functors.

Proposition 3.3. Let $k$ contain $\mathbb{Q}$ and $A$ be an algebra. Then there is an isomorphism

$$
L_{n}^{\mathcal{F}} \mathcal{A} b(A) \cong L_{n-1}^{\mathcal{F}}(-\otimes-)(A), \quad n \geqslant 2
$$

and an exact sequence

$$
0 \longrightarrow L_{1}^{\mathcal{F}} \mathcal{A} b(A) \longrightarrow A \otimes A \longrightarrow A \longrightarrow \mathcal{A} b(A) \longrightarrow 0 .
$$

Proof. Consider the $\mathcal{F}$-cotriple resolution $\left(\mathcal{F}_{*}(A), d_{0}^{0}, A\right)$ of $A$. Applying the exact sequence $(2)$ dimensionwise to the simplicial algebra $\mathcal{F}_{*}(A)$ and using the fact that $H C_{1}\left(\mathcal{F}_{n}(A)\right)=0, n \geqslant 0$, we obtain the following short exact sequence of simplicial modules:

$$
0 \longrightarrow \mathcal{F}_{*}(A) \otimes \mathcal{F}_{*}(A) \stackrel{\mu}{\longrightarrow} \mathcal{F}_{*}(A) \longrightarrow \mathcal{A} b\left(\mathcal{F}_{*}(A)\right) \longrightarrow 0 .
$$

Since $H_{n}\left(\mathcal{F}_{*}(A)\right)=0, n \geqslant 1$, Lemma 3.2 and the induced long exact homology sequence clearly complete the proof.

Corollary 3.4. Let $k$ contain $\mathbb{Q}$ and $A$ be an algebra. Then there is an isomorphism

$$
H C_{1}(A) \cong L_{1}^{\mathcal{F}} \mathcal{A} b(A)
$$

Proof. Straightforward from Proposition 3.3 and (2).

Note that this isomorphism between the first cyclic homology and the first derived functor of the additive abelianisation functor holds without any restriction on the algebra $A$. But in higher dimensions we need an extra condition on $A$ to prove the same result. Namely, we have the following. 
Theorem 3.5. Let $k$ contain $\mathbb{Q}$ and $A$ be an algebra, which is flat as a module. Then there is an isomorphism

$$
H C_{n}(A) \cong L_{n}^{\mathcal{F}} \mathcal{A} b(A), \quad n \geqslant 0 .
$$

Proof. We only have to show that the functor $\Phi: \mathbf{A l g} \rightarrow \mathbf{C o m p}_{\geqslant 0}$, defined by $\Phi(A)$ $=\operatorname{Tot}(C C(A))_{*}$ for $A \in \mathbf{A l g}$, satisfies conditions (i) and (ii) of Proposition 2.2.

Thanks to Lemma 2.1, it is easy to see that the augmented simplicial module

$$
\left(\operatorname{Tot}(C C(A))_{n}\left(\mathcal{F}_{*}(A)\right), \operatorname{Tot}(C C(A))_{n}\left(d_{0}^{0}\right), \operatorname{Tot}(C C(A))_{n}(A)\right), n \geqslant 0
$$

is acyclic, where $\left(\mathcal{F}_{*}(A), d_{0}^{0}, A\right)$ is the $\mathcal{F}$-cotriple resolution of $A$. Condition (i) is fulfilled.

The fulfilment of condition (ii) follows from the fact that there is an equality $H C_{n}(\mathcal{F}(A))=0, n \geqslant 1$, for any algebra $A$ over $k$ containing $\mathbb{Q}[\mathbf{1 7}]$.

Remark 3.6.

(i) Theorem 3.5 generalises the result of Feigin and Tsygan [11] given for algebras over a field of characteristic zero;

(ii) Let $k$ contain $\mathbb{Q}$ and $F$ be a free algebra. Then Connes' Periodicity Exact Sequence implies that there is a natural isomorphism

$$
\mathcal{A} b(F) \stackrel{B}{\cong} H H_{1}(F) .
$$

Therefore for a given algebra $A$ we have the isomorphism

$$
L_{n}^{\mathcal{F}} \mathcal{A} b(A) \cong L_{n}^{\mathcal{F}} H H_{1}(A), \quad n \geqslant 0 .
$$

Using Theorem 3.5 it is easy to check that, in case $A$ is flat as a module, the long exact sequence of Proposition 3.1 turns into Connes' Periodicity Exact Sequence.

Now let $A$ denote an algebra and $m \geqslant 1$. The general linear Lie algebra $\operatorname{gl}_{m}(A)$ is the Lie algebra of $m \times m$ matrices with entries in $A$, while the special linear Lie algebra $\operatorname{sl}_{m}(A)$ is the Lie algebra of $m \times m$ matrices of trace zero (the trace being evaluated in $A /[A, A])$. It is clear that $\operatorname{sl}_{m}(A)$ is an ideal in $\mathrm{gl}_{m}(A)$, and there is the following short exact sequence of Lie algebras:

$$
0 \longrightarrow \operatorname{sl}_{m}(A) \longrightarrow \mathrm{gl}_{m}(A) \longrightarrow A /[A, A] \longrightarrow 0
$$

(see [16, Chapter 10]).

Corollary 3.7. Let $k$ contain $\mathbb{Q}, A$ be an algebra, which is flat as a module, and $m \geqslant 1$. Then there is an isomorphism

$$
H C_{n+1}(A) \cong L_{n}^{\mathcal{F}} \operatorname{sl}_{m}(A), \quad n \geqslant 1 .
$$

Proof. Thanks to Lemma 2.1(i), it is easy to see that the augmented simplicial module $\left(\operatorname{gl}_{m}\left(\mathcal{F}_{*}(A)\right), \mathrm{gl}_{m}\left(d_{0}^{0}\right), \mathrm{gl}_{m}(A)\right)$ is acyclic for the $\mathcal{F}$-cotriple resolution $\left(\mathcal{F}_{*}(A), d_{0}^{0}, A\right)$ of the algebra $A$. Hence Theorem 3.5 and the long exact homology sequence, induced by the short exact sequence of complexes obtained by applying $(3)$ to $\left(\mathcal{F}_{*}(A), d_{0}^{0}, A\right)$, imply the result. 
As an application of Theorem 3.5 we give an axiomatic description of cyclic homology of algebras.

Let $\mathcal{L}$ be a full subcategory of the category Alg consisting of all algebras, which are flat as modules. It is easy to see that $\mathcal{F}(L) \in \mathcal{L}$ for $L \in \mathcal{L}$ (cf. Lemma 2.1). Then we have the following.

Proposition 3.8. Let $k$ contain $\mathbb{Q}$ and $\Phi: \mathbf{A l g} \rightarrow \mathbf{C o m p}_{\geqslant 0}$ be a functor satisfying the following axioms:

(a) the augmented simplicial module $\left(\Phi_{n}\left(\mathcal{F}_{*}(L)\right), \Phi_{n}\left(d_{0}^{0}\right), \Phi_{n}(L)\right)$ is acyclic for $L \in \mathcal{L}$ and $n \geqslant 0$, where $\left(\mathcal{F}_{*}(L), d_{0}^{0}, L\right)$ is the $\mathcal{F}$-cotriple resolution of $L$;

(b) $H_{n}^{\Phi}(\mathcal{F}(L))=0$ for $L \in \mathcal{L}$ and $n>0$;

(c) there is a natural isomorphism $H_{0}^{\Phi}(L) \cong H C_{0}(L)$ for $L \in \mathcal{L}$.

Then there is a natural isomorphism $H_{n}^{\Phi}(L) \cong H C_{n}(L)$ for $L \in \mathcal{L}$ and $n \geqslant 0$.

Proof. By (c), we have the isomorphism of augmented simplicial modules

$$
\left(H_{0}^{\Phi}\left(\mathcal{F}_{*}(L)\right), H_{0}^{\Phi}\left(d_{0}^{0}\right), H_{0}^{\Phi}(L)\right) \cong\left(H C_{0}\left(\mathcal{F}_{*}(L)\right), H C_{0}\left(d_{0}^{0}\right), H C_{0}(L)\right) .
$$

Thus there is a natural isomorphism

$$
L_{n}^{\mathcal{F}} H_{0}^{\Phi}(L) \cong L_{n}^{\mathcal{F}} H C_{0}(L) \quad \text { for } \quad L \in \mathcal{L} \text { and } n \geqslant 0 .
$$

Since the functor $\Phi: \mathbf{A l g} \rightarrow \mathbf{C o m p}_{\geqslant 0}$ satisfy the axioms (a) and (b), it follows from Proposition 2.2 that we have a natural isomorphism

$$
H_{n}^{\Phi}(L) \cong L_{n}^{\mathcal{F}} H_{0}^{\Phi}(L) \quad \text { for } \quad L \in \mathcal{L} \text { and } n \geqslant 0 .
$$

Hence Theorem 3.5 completes the proof.

Another application of Theorem 3.5 is to obtain the generalised Hopf type formulas for the cyclic homology of algebras, using the method of $n$-fold Cech derived functors $[\mathbf{7}, \mathbf{1 3}]$. We can think as an initial result in this direction the exact sequence

$$
H C_{1}(F) \longrightarrow H C_{1}(A) \longrightarrow I /[I, F] \longrightarrow F /[F, F] \longrightarrow A /[A, A] \longrightarrow 0
$$

(see [19]), where $F \stackrel{\alpha}{\rightarrow} A$ is a surjective homomorphism of algebras and $I=\operatorname{Ker} \alpha$. In case $\alpha$ is a free presentation of the algebra $A$, then (4) induces the Hopf formula for the first cyclic homology

$$
H C_{1}(A) \cong(I \cap[F, F]) /[I, F] .
$$

We generalise formula (5) to any dimension.

Now let $n$ be a non-negative integer and let $\mathcal{C}_{n}$ denote the category determined by the ordered set of all subsets of the set $\langle n\rangle=\{1, \ldots, n\}$. An $n$-cube of algebras is a functor $\mathfrak{F}: \mathcal{C}_{n} \rightarrow \mathbf{A l g}$, and we will denote its component parts by $X \mapsto \mathfrak{F}_{X}, \rho_{Y}^{X} \mapsto \alpha_{Y}^{X}$. 
Example 3.9. Let $\left(A_{*}, d_{0}^{0}, A\right)$ be an augmented simplicial algebra. A natural $n$-cube of algebras $A^{(n)}: \mathcal{C}_{n} \rightarrow \mathbf{A l g}, n \geqslant 1$ is defined in the following way:

$$
A_{A}^{(n)}=A_{n-1-|X|} \quad \text { for all } X \subseteq\langle n\rangle
$$

and

$$
\alpha_{X \cup\{j\}}^{X}=d_{k-1}^{n-1-|X|} \quad \text { for all } X \neq\langle n\rangle, j \notin X,
$$

where $A_{-1}=A, \delta(k)=j$ and $\delta:\langle n-|X|\rangle \rightarrow\langle n\rangle \backslash X$ is the unique monotone bijection.

Given an $n$-cube of algebras $\mathfrak{F}$, it is easy to see there exists a natural homomorphism $\mathfrak{F}_{X} \stackrel{\alpha_{X}}{\longrightarrow} \lim _{Y \supset X} \mathfrak{F}_{Y}$ for each $X \subseteq\langle n\rangle, X \neq\langle n\rangle$.

Let $A$ denote an algebra. An $n$-cube of algebras $\mathfrak{F}$ is called an $n$-presentation of the algebra $A$ if $\mathfrak{F}_{\langle n\rangle}=A$. An $n$-presentation $\mathfrak{F}$ of $A$ is called free if the algebra $\mathfrak{F}_{X}$ is free for each $X \neq\langle n\rangle$ and called exact if the homomorphism $\alpha_{X}$ has a $k$-linear splitting for each $X \neq\langle n\rangle$.

Let $\left(F_{*}, d_{0}^{0}, A\right)$ be an augmented simplicial algebra. It is easy to check that $\left(F_{*}, d_{0}^{0}, A\right)$ is a free simplicial resolution of $A$ if and only if the $n$-cube of algebras $F^{(n)}$ is a free exact $n$-presentation of $A$ for any $n \geqslant 1$. Clearly, this fact ensures the existence of free exact $n$-presentations of algebras. Note also that one can construct a free exact $n$-presentation of an algebra step-by-step similarly to a fibrant $n$-presentation of a group in the sense of Brown-Ellis [5].

Theorem 3.10 (Hopf type formulas for cyclic homology). Let $k$ contain $\mathbb{Q}, A$ be an algebra, which is flat as a module and $\mathfrak{F}$ a free exact $n$-presentation of $A$. Then there is an isomorphism

$$
H C_{n}(A) \cong\left(\underset{i \in\langle n\rangle}{\cap} I_{i} \cap[F, F]\right) /\left(\sum_{X \subseteq\langle n\rangle}\left[{ }_{i \in X} I_{i}, \underset{i \notin X}{\cap} I_{i}\right]\right), \quad n \geqslant 1,
$$

where $\left(F ; I_{1}, I_{2}, \ldots, I_{n}\right)$ is the normal $(n+1)$-ad of algebras induced by $\mathfrak{F}$ in the following way: $F=\mathfrak{F}_{\emptyset}$ and $I_{i}=\operatorname{Ker} \alpha_{\{i\}}^{\emptyset}, i \in\langle n\rangle$.

Proof. Using the method of $n$-fold Čech derived functors, the proof is similar to that given in $[\mathbf{6}, \mathbf{7}]$ and is left to the reader. For the sketch of the proof see [14].

\section{Periodic and negative cyclic homologies via derived func- tors}

In this section, continuing the lines of the previous one, we describe the periodic cyclic and negative cyclic homologies of algebras in terms of cotriple derived functors.

We begin by recalling the definitions of these homology theories. Given an algebra $A$, let $C N(A)$ denote the negative cyclic homology bicomplex, obtained from $C P(A)$ by deleting all columns whose indices are $\geqslant 2$. The homology groups of the complexes $\operatorname{Tot}(C P(A))_{*}$ and $\operatorname{Tot}(C N(A))_{*}$ define, respectively, the periodic cyclic homology, $H P_{*}(A)$, and the negative cyclic homology, $H N_{*}(A)$, of the algebra $A$. 
Now, for any $n \geqslant 0$, we define a functor

$$
B_{n}: \mathbf{A l g} \longrightarrow \operatorname{Mod} \quad \text { by } B_{n}(A)=\operatorname{Tot}(C C(A))_{n} / \operatorname{Im} \partial_{n+1}, \quad A \in \mathbf{A l g}
$$

where $\partial_{n+1}$ is the boundary operator of $\operatorname{Tot}(C C(A))_{*}$. It is well known that there is a natural transformation of functors $\operatorname{Tot}(C C)_{n+2} \longrightarrow \operatorname{Tot}(C C)_{n}, n \geqslant 0$, inducing Connes' periodicity operator $S: H C_{n+2} \rightarrow H C_{n}, n \geqslant 0$. Hence there is a natural transformation $B_{n+2} \longrightarrow B_{n}, n \geqslant 0$, implying the inverse system of functors $\left\{B_{n}, n\right.$ is even $\}$ and $\left\{B_{n}, n\right.$ is odd $\}$.

$$
B_{\text {even }}^{\infty}: \mathbf{A l g} \longrightarrow \text { Mod and } B_{\text {odd }}^{\infty}: \text { Alg } \longrightarrow \text { Mod, }
$$

respectively, by the formulas

$$
B_{\text {even }}^{\infty}(A)=\lim _{k} B_{2 k}(A) \quad \text { and } \quad B_{\text {odd }}^{\infty}(A)=\underbrace{\lim }_{k} B_{2 k+1}(A)
$$

for $A \in \mathbf{A l g}$.

For a given algebra $A$, consider truncated complexes of the periodic cyclic homology complex Tot $(C P(A))_{*}$ :

$$
\begin{aligned}
& \operatorname{Tot}_{\geqslant 0}(C P(A))_{*} \equiv \cdots \stackrel{\partial_{\text {odd }}}{\longrightarrow} \prod_{n \geqslant 0} A^{\otimes n+1} \stackrel{\partial_{\text {even }}}{\longrightarrow} \prod_{n \geqslant 0} A^{\otimes n+1} \stackrel{\partial_{\text {odd }}}{\longrightarrow} \prod_{n \geqslant 0} A^{\otimes n+1}, \\
& \operatorname{Tot}_{\geqslant 1}(C P(A))_{*} \equiv \cdots \stackrel{\partial_{\text {even }}}{\longrightarrow} \prod_{n \geqslant 0} A^{\otimes n+1} \stackrel{\partial_{\text {odd }}}{\longrightarrow} \prod_{n \geqslant 0} A^{\otimes n+1} \stackrel{\partial_{\text {even }}}{\longrightarrow} \prod_{n \geqslant 0} A^{\otimes n+1},
\end{aligned}
$$

where the even, $\partial_{\text {even }}$, and the odd, $\partial_{\text {odd }}$, boundaries are total boundaries of $C P(A)$ induced by $\left(1-t,-b^{\prime}\right)$ and $(N, b)$.

It is clear that we have the equalities

$$
\begin{aligned}
& H_{0}\left(\operatorname{Tot}_{\geqslant 0}(C P(A))_{*}\right)=\prod_{n \geqslant 0} A^{\otimes n+1} / \operatorname{Im} \partial_{\text {odd }}, \quad H_{i}\left(\operatorname{Tot}_{\geqslant 0}(C P(A))_{*}\right)=H P_{i}(A), i \geqslant 1, \\
& \text { and } \\
& H_{0}\left(\operatorname{Tot}_{\geqslant 1}(C P(A))_{*}\right)=\prod_{n \geqslant 0} A^{\otimes n+1} / \operatorname{Im} \partial_{\text {even }}, \quad H_{i}\left(\operatorname{Tot}_{\geqslant 1}(C P(A))_{*}\right)=H P_{i+1}(A), i \geqslant 1 .
\end{aligned}
$$

Lemma 4.1. Let $k$ contain $\mathbb{Q}$ and $F$ be a free algebra. Then there are natural isomorphisms

$$
B_{\text {even }}^{\infty}(F) \cong \prod_{n \geqslant 0} F^{\otimes n+1} / \operatorname{Im} \partial_{\text {odd }} \quad \text { and } \quad B_{\text {odd }}^{\infty}(F) \cong \prod_{n \geqslant 0} F^{\otimes n+1} / \operatorname{Im} \partial_{\text {even }}
$$

Proof. We shall prove only the first isomorphism. The proof of the second is similar and will be omitted.

In fact, by definition

$$
B_{\text {even }}^{\infty}(F)=\underbrace{\lim }_{n} B_{2 n}(F)=\underbrace{\lim }_{n}\left(\operatorname{Tot}(C C(F))_{2 n} / \operatorname{Im} \partial_{2 n+1}\right) .
$$

Since the natural homomorphism $\operatorname{Im} \partial_{2 n+1} \rightarrow \operatorname{Im} \partial_{2 n-1}$ is surjective for any $n \geqslant 1$, we have $\underset{n}{\lim _{n}^{(1)}}\left(\operatorname{Im} \partial_{2 n+1}\right)=0$ where ${\underset{n}{n}}^{\lim }(1)$ is the first right derived functor of $\lim ^{\lim }$. 
This implies there is a natural isomorphism

$$
B_{\text {even }}^{\infty}(F) \cong \bigsqcup_{n}^{\lim }\left(\operatorname{Tot}(C C(F))_{2 n}\right) / \overleftarrow{n}_{n}^{\lim }\left(\operatorname{Im} \partial_{2 n+1}\right) .
$$

It is easy to see that

$$
\lim _{n}\left(\operatorname{Tot}(C C(F))_{2 n}\right) \cong \prod_{n \geqslant 0} F^{\otimes n+1} .
$$

Therefore it remains to show that we have an isomorphism

$$
{\underset{\lim }{n}}_{n}\left(\operatorname{Im} \partial_{2 n+1}\right) \cong \operatorname{Im} \partial_{\text {odd }} \text {. }
$$

In fact, we have equalities $H C_{2 n}(F)=0$ and $H P_{n}(F)=0, n \geqslant 1$. Hence $\operatorname{Im} \partial_{2 n+1}$ $=\operatorname{Ker} \partial_{2 n}, n \geqslant 1$ and $\operatorname{Im} \partial_{\text {odd }}=\operatorname{Ker} \partial_{\text {even }}$. One readily checks that

$$
\underset{n}{\lim } \operatorname{Ker} \partial_{2 n} \cong \operatorname{Ker} \partial_{\text {even }}
$$

and the result follows.

Theorem 4.2. Let $k$ contain $\mathbb{Q}$ and $A$ be an algebra, which is flat as a module. Then there are natural isomorphisms

$$
H P_{n}(A) \cong L_{n}^{\mathcal{F}} B_{\text {even }}^{\infty}(A) \quad \text { and } \quad H P_{n+1}(A) \cong L_{n}^{\mathcal{F}} B_{\text {odd }}^{\infty}(A), \quad n \geqslant 1 .
$$

Proof. Using Lemma 4.1, we only have to show that the functors $\Phi, \Psi: \mathbf{A l g} \rightarrow$ Comp $_{\geqslant 0}$, defined by $\Phi(A)=\operatorname{Tot}_{\geqslant 0}(C P(A))_{*}$ and $\Psi(A)=\operatorname{Tot}_{\geqslant 1}(C P(A))_{*}$ for $A \in \mathbf{A l g}$, satisfy conditions (i) and (ii) of Proposition 2.2.

Condition (i) is fulfilled, since by Lemma 2.1 the augmented simplicial module

$$
\prod_{n \geqslant 0} \mathcal{F}_{*}(A)^{\otimes n+1} \rightarrow \prod_{n \geqslant 0} A^{\otimes n+1}
$$

is acyclic, where $\left(\mathcal{F}_{*}(A), d_{0}^{0}, A\right)$ is the $\mathcal{F}$-cotriple resolution of $A$.

The fulfilment of condition (ii) follows from the fact that there is an equality $H P_{*}(\mathcal{F}(A))=0$ for any algebra $A$ over $k$ containing $\mathbb{Q}$.

Now we look at negative cyclic homology $H N_{*}$ in positive dimensions. Note that, in non-positive dimensions, the negative cyclic homology coincides with the periodic cyclic homology and is already described as cotriple derived functors.

Given an algebra $A$, consider the natural surjective homomorphism of modules

$$
B_{\text {even }}^{\infty}(A) \rightarrow B_{0}(A)=A /[A, A] .
$$

Define the functor

$$
B^{-}: \mathbf{A l g} \longrightarrow \mathbf{M o d} \text { given by } B^{-}(A)=\operatorname{Ker}\left\{B_{\text {even }}^{\infty}(A) \rightarrow B_{0}(A)\right\} .
$$

Theorem 4.3. Let $k$ contain $\mathbb{Q}$ and $A$ be an algebra, which is flat as a module. Then there is a natural isomorphism

$$
H N_{n+2}(A) \cong L_{n}^{\mathcal{F}} B^{-}(A), \quad n \geqslant 1
$$


Proof. It is clear that we have the short exact sequence of complexes

$$
0 \longrightarrow \operatorname{Tot}_{\geqslant 2}(C N(A))_{*} \longrightarrow \operatorname{Tot}_{\geqslant 2}(C P(A))_{*} \longrightarrow \operatorname{Tot}(C C(A))_{*} \longrightarrow 0
$$

and the equality $H_{n}\left(\operatorname{Tot}_{\geqslant 2}(C N(A))\right)=H N_{n+2}(A), n \geqslant 1$. Moreover, the functor $\Phi: \mathbf{A l g} \rightarrow \mathbf{C o m p}_{\geqslant 0}$, defined by $\Phi(A)=\operatorname{Tot}_{\geqslant 2}(C N(A))_{*}, A \in \mathbf{A l g}$, satisfies the conditions (i) and (ii) of Proposition 2.2. Hence there is a natural isomorphism

$$
L_{n}^{\mathcal{F}} H_{0}\left(\operatorname{Tot}_{\geqslant 2}(C N(A))_{*}\right) \cong H N_{n+2}(A), \quad n \geqslant 1 .
$$

Thus, to finish the proof, it suffices to show that $H_{0}\left(\operatorname{Tot}_{\geqslant 2}(C N(F))_{*}\right)$ is naturally isomorphic to $B^{-}(F)$ for any free algebra $F$. But we have $H C_{n}(F)=H P_{n}(F)$ $=H N_{n+1}(F)=0$ for $n \geqslant 1$. Clearly the long exact homology sequence induced by the short exact sequence $(6)$ gives the short exact sequence of modules

$$
0 \longrightarrow H_{0}\left(\operatorname{Tot}_{\geqslant 2}(C N(F))_{*}\right) \longrightarrow H_{0}\left(\operatorname{Tot}_{\geqslant 2}(C P(F))_{*}\right) \longrightarrow H C_{0}(F) \longrightarrow 0 .
$$

Hence, by Lemma 4.1, we obtain the natural short exact sequence of modules

$$
0 \longrightarrow H_{0}\left(\operatorname{Tot}_{\geqslant 2}(C N(F))_{*}\right) \longrightarrow B_{\text {even }}^{\infty}(F) \longrightarrow F /[F, F] \longrightarrow 0,
$$

which completes the proof.

\section{References}

[1] M. Barr, Shukla cohomology and triples, J. Algebra 5 (1967), 222-231.

[2] M. Barr, Cartan-Eilenberg cohomology and triples, J. Pure Appl. Algebra 112 (1996), no. 3, 219-238.

[3] M. Barr and J. Beck, Acyclic modules and triples, Proc. conference on categorical algebra (La Jolla 1965), Springer-Verlag, New York (1966), 336-343.

[4] M. Barr and J. Beck, Homology and standard constructions, in: (B. Eckmann, ed.), Seminar on triples and categorical homology theory, Lecture Notes in Math. 80, Springer-Verlag, New York (1969), 245-335.

[5] R. Brown and G.J. Ellis, Hopf formulae for the higher homology of a group, Bull. London Math. Soc. 20 (1988), no. 2, 124-128.

[6] J.M. Casas, N. Inassaridze, E. Khmaladze and M. Ladra, Homology of $(n+1)-$ types and Hopf type formulas, J. Pure Appl. Algebra 200 (2005), no. 3, 267280.

[7] G. Donadze, N. Inassaridze and T. Porter, $N$-fold Čech derived functors and generalised Hopf type formulas, K-Theory 35 (2005), no. 3-4, 341-373.

[8] J. Duskin, Simplicial methods and the interpretation of "triple" cohomology, Mem. Amer. Math. Soc. 163, no. 2, 1975.

[9] G.J. Ellis, Relative derived functors and the homology of groups, Cahier Topologie et Géometrie Diff. Cat. 31 (1990), no. 2, 121-135.

[10] G.J. Ellis and R. Mikhailov, A colimit of classifying spaces, Advances in Math. 223 (2010), no. 6, 2097-2113.

[11] B.L. Feigin and B.L. Tsygan, Additive $K$-theory, in: $K$-theory, arithmetic and geometry (Moscow, 1984-1986), 67-209, Lecture Notes in Math. 1289, Springer-Verlag, New York, 1987. 
[12] H. Inassaridze, Non-abelian homological algebra and its applications, Math. Appl. 421, Kluwer Academic Publishers, Dordrecht, 1997.

[13] N. Inassaridze, $N$-fold Čech derived functors of group valued functors, Bull. Georgian Acad. Sci. 168 (2003), no. 2, 223-226.

[14] N. Inassaridze and M. Ladra, Hopf type formulas for cyclic homology, C. R. Acad. Sci. Paris, Ser. I 346 (2008), 385-390.

[15] F. Keune, Derived functors and algebraic $K$-theory, in: (H. Bass, ed.), Algebraic K-theory I. Higher K-theories, Lecture Notes in Math. 341 (1973), SpringerVerlag, New York, 166-176.

[16] J.-L. Loday, Cyclic homology, Grundlehren der Mathematischen Wissenschaften, 301, Springer-Verlag, New York, 1992.

[17] J.-L. Loday and D.G. Quillen, Cyclic homology and the Lie algebra homology of matrices, Comment. Math. Helv. 59 (1984), no. 4, 565-591.

[18] D. Quillen, Spectral sequences of a double semi-simplicial group, Topology 5 (1966), 155-157.

[19] D. Quillen, Cyclic cohomology and algebra extensions, K-Theory 3 (1989), no. 3, 205-246.

[20] R.G. Swan, Some relations between higher K-functors, J. Algebra 21 (1972), $113-136$.

[21] C.A. Weibel, An introduction to homological algebra, Camb. Stud. Adv. Math. 38, Camb. Univ. Press, Cambridge, 1994.

Guram Donadze donad@rmi.acnet.ge

Department of Algebra, A. Razmadze Mathematical Institute, M. Alexidze St. 1, 0193 Tbilisi, Georgia \& Tbilisi Centre for Mathematical Sciences, Georgia

Nick Inassaridze niko.inas@gmail.com

Department of Algebra, A. Razmadze Mathematical Institute, M. Alexidze St. 1, 0193 Tbilisi, Georgia \& Tbilisi Centre for Mathematical Sciences, Georgia Departamento de Matemática Aplicada I, Universidad de Vigo, EUIT Forestal, 36005 Pontevedra, Spain

Manuel Ladra manuel.ladra@usc.es

Departamento de Álgebra, Universidad de Santiago de Compostela, 15782 Santiago de Compostela, Spain 